\title{
El suicidio infantil, retos de una sociedad global. Tópicos del Trabajo Social
}

\section{Child suicide, challenges of a global society.Topics of Social Work}

\author{
JESÚS ACEVEDO ALEMÁN \\ Doctor en Trabajo Social. Facultad en Trabajo Social. Universidad Autónoma de Coahuila. Saltillo, Coahuila, México. \\ jesus_acevedo@uadec.edu.mx.https://orcid.org/0000-0001-6089-9132
}

Recibido: 24/07/2019

Aceptado: 17/10/2019

\begin{abstract}
Resumen: Cada año se suicidan casi un millón de personas, lo que supone una tasa de mortalidad global de 16 por 100,000 habitantes; dicho de otra manera, se presenta una muerte cada 40 segundos. Representando el suicidio una de las tres primeras causas de defunción entre las personas de 15 a 44 años en algunos países, y la segunda causa en el grupo de 10 a 24 años (Organizción Mundial de la Salud, 2018). Aunque tradicionalmente las mayores tasas de suicidio se han registrado entre personas mayores de 18 años, las tasas entre los jóvenes y niños han ido en aumento hasta el punto de que ahora estos son el grupo de mayor riesgo en un tercio de los países, tanto en el mundo desarrollado, como en el mundo en desarrollo. En tal sentido, el presente texto a partir de una revisión documental y de fuentes oficiales, contribuye en la reflexión y análisis de este fenomeno, particularmente en el suicidio infantil, el cual se consideraba anteriomente "poco frecuente" (Durkheim, 1965); pero en la actualidad, se ha ido incrementando en México, lo cual requiere de la suma de esfuerzos para su prevención y atención, pero sobredo comvoca a la participacion de los trabajadores sociales desde su quehacer profesional para generar estrategias y contribuir de una manera protagonica frente a dicho fenomeno. Concluyendo con algunas recomendaciones y propuestas desde dicha trichera disciplinar.
\end{abstract}

Palabras claves: suicidio; suicidio en México, suicidio infantil

Summary: Nearly one million people commit suicide each year, which represents a global mortality rate of 16 per 100,000 inhabitants; In other words, a death occurs every 40 seconds. Representing suicide one of the first three causes of death among people aged 15 to 44 in some countries, and the second cause in the group of 10 to 24 years (World Health Organization, 2018). Although traditionally the highest suicide rates have been registered among people over 18 , the rates among young people and children have been rising to the point that these are now the highest risk group in a third of the countries, both in the developed world, as in the developing world. In this sense, the present text, based on a documentary review and official sources, contributes to the reflection and analysis of this phenomenon, particularly in child suicide, which was previously considered "infrequent" (Durkheim,1965); but At present, it has been increasing in Mexico, which requires the sum of efforts for its prevention and care, but above all it invokes the participation of social workers from their professional work to generate strategies and contribute in a leading way against said phenomenon, concluding with some recommendations and proposals from said disciplinary trichera.

Keywords: suicide; suicide in Mexico, child suicide. 


\section{EL SUICIDIO, IDEAS CENTRALES}

En la obra el Comportamiento Suicida de Quintanar (2007) se afirma que el término "suicidio" surge en el siglo XVII en gran Bretaña y en Francia en el siglo XVIII presentando su origen con el Abate ${ }^{1}$ Prevost en 1734, término que posteriormente es retomado por el Abate Desfontaines en 1737; incluido en 1762 en la académia francesa de la lengua, definido como "acto del que se mata a sí mismo", el mismo autor destaca que el suicidio, se percibe como un acto ante el cual, el individuo se encuentra vulnerable desde tiempos históricos; problemática no nueva que va en aumento al paso de los años.

Reyes (1991) sostiene que el suicidio representa una realidad humana, que ha existido desde que hay vida en el planeta, ejemplificando que en la Biblia se narran los primeros actos suicidas dentro de los pasajes bíblicos, donde se dan a conocer la muerte de algunos personajes bajo condiciones de autolesiones como es el caso de Judas discípulo de Jesús, el cual no tolero haber traicionado a su maestro y decide poner fin a su vida ahorcándose (Mateo 27: 3-10); o bien, la historia de Samson juez de Israel (Jueces 16:30); o la muerte de Abimelek (Jueces 9:54); Saúl y su escudero (1 Samuel 31: $4,5)$ y seguramente si se analiza a profundidad dicho texto se podrían encontrar más sucesos similares.

En la Europa Antigua, sobre todo durante el Imperio Romano, el suicidio se consentía e incluso era considerado un acto honroso. El filósofo romano Séneca lo ensalzaba como el último acto de una persona libre. Sin embargo para San Agustín, el suicidio era un pecado. Varios de los primeros concilios de la Iglesia cristiana decretaron que no se celebraría ningún funeral por ninguna persona que se hubiera suicidado. En las legislaciones medievales se ordenaba la confiscación de todas las propiedades del suicida y el cadáver sufría todo tipo de humillaciones. Actualmente, el suicidio, está condenado en las religiones cristiana, judía e islámica (Maldonado, 2003).

Durkheim (1965) postulaba que el suicidio era un fenómeno de explicaciones sociológicas, resultado de una falta de integración del individuo en la sociedad, más que un puro acto individualista, donde las sociedades modernas mantienen niveles de suicidio muy elevados, producto, en parte, de la permisividad social y del considerable grado de tolerancia. Considerando el mismo que, el suicidio representa todo caso de muerte que resulte directa o indirectamente de un acto positivo o negativo, ejecutado por la propia víctima, a sabiendas de que habría de producir este resultado.

Actualmente el suicidio se puede entender como un proceso, complejo y dinámico, que según Chávez, Medina y Macías
(2008) está conformado por varias etapas que comienzan con una ideación suicida pasiva, seguida por fases cada vez más activas como la visualización del propio acto y su manifestación mediante amenazas verbales y gestos suicidas, la planeación y preparación, la ejecución del intento y por último el suicidio consumado. Argumentando los mismos que, la persona que tiene pensamientos suicidas es más propensa a intentar quitarse la vida, así como quien lo ha intentado una vez, está más propenso a intentarlo nuevamente en menos de un año, y ahora con mayores probabilidades de éxito; la estrecha relación entre ideación y conducta suicida no sólo ha sido verificada en adultos sino también en adolescentes y niños.

\subsection{El suicidio en el mundo}

Investigadores como García, Contreras y Orozco (2006) describen que en la actualidad, se presenta un incremento considerable en los registros de muertes por esta causa, indicando que en el año de 1980 se cometieron 672 suicidios; años más tarde en 1990 aumentó a 1,405 casos; mientras que en el 2002 alcanzaron los 3,089 suicidios. Destacando que, en el 2000 se suicidaron casi un millón de personas en el mundo, lo que convirtió al suicidio en la décimo tercera causa de muerte.

La Organización Mundial de la Salud (2012; Sinembargo.mx, 2013) estima que para el 2020 las víctimas podrían ascender a 1.5 millones por año. En tal sentido García et al. (2006) consideran que típicamente el 25\% de quienes intentaron una vez el suicidio, lo intentarán nuevamente el próximo año y, el 10\% lo logrará en un plazo de diez años, señalando que entre el $45 \%$ y el $70 \%$ de quienes intentan el suicidio sufren principalmente de depresión, con rasgos de impulsivilidad y agresividad, trastornos de la personalidad y problemas de alcoholismo o algún tipo de adicción, que a menudo van unidos a una pérdida reciente o a problemas de conducta (Público ES, 2008).

Ante dicho escenario el Centro de Información de las Naciones Unidas [CINU] (2013) sostiene que, cada día hay en promedio casi 3,000 personas ponen fin a su vida, y al menos veinte personas intentan suicidarse por cada una que lo consigue. Según la Organización Mundial de la Salud (2014) las estimaciones específicas por países de las tasas de suicidio en 2012 de los 172 Estados miembros con 300,000 habitantes o más se presentan en los países con poblaciones más pequeñas.

\subsection{El suicidio en México}

En México de manera particular, se presenta una cifra obscura del suicidio, dicho dato se oculta detrás de 


\section{TABLA I. InTentos de SUICIDio y SUICIDIOS en México 2005}

\begin{tabular}{|c|c|c|c|c|c|c|c|c|c|}
\hline & Totalw & Amorosa & $\begin{array}{l}\text { Dificultades } \\
\text { Económicos }\end{array}$ & $\begin{array}{l}\text { Disgusto } \\
\text { familiar }\end{array}$ & $\begin{array}{c}\text { Enfermedad } \\
\text { grave } \\
\text { incurable }\end{array}$ & $\begin{array}{l}\text { Enfermedad } \\
\text { mental }\end{array}$ & Remordimiento & Otra & Se ignora \\
\hline $\begin{array}{l}\text { Intentos de } \\
\text { suicidio }\end{array}$ & 193 & 26 & 12 & 55 & 5 & 13 & 0 & 20 & 62 \\
\hline Hombres & 91 & 9 & 9 & 23 & 2 & 4 & 0 & 12 & 32 \\
\hline Mujeres & 102 & 17 & 3 & 32 & 3 & 9 & 0 & 8 & 30 \\
\hline Suicidios & 3324 & 230 & 94 & 280 & 172 & 141 & 33 & 287 & 2087 \\
\hline Hombres & 2753 & 186 & 81 & 220 & 141 & 106 & 31 & 234 & 1754 \\
\hline Mujeres & 571 & 44 & 13 & 60 & 31 & 35 & 2 & 53 & 33 \\
\hline
\end{tabular}

Fuente: INEGI (2005)

los registros oficiales; de un acto suicida, hay muchos más que no son registrados, ya sea por cuestiones familiares, o por motivos de tabú o religiosos (Nadelsticher y Gorenc, 1985). Por ejemplo, en el año de 1970 se registraron 554 defunciones por suicidio en el interior de la república mexicana, y en 1994 se registraron 2,603; durante dicho periodo la tasa de muertes por suicidio paso de 1.13 por cada 100,000 habitantes en 1970 a 2.89 por cada 100,000 habitantes en el año de 1994 (Borges, Orozco, Benjet y Medina, 2010).

Por su parte, el Instituto Nacional de Estadística Geografía e Informática (INEGI) (2005) reportó un total de 3,324 suicidios en México, de los cuales se destacó como principal causa de muerte: por un disgusto familiar (280 suicidios); seguida de por cuestiones amorosas (230 suicidios); y finalmente por enfermedad grave e incurable (172 casos) (tabla I). Por su parte, la Cédula Forense del Sistema de Vigilancia Epidemiológica de las Adicciones (SISVEA) (Ocampo, Bojorquez y Cortés, 2009) describe que para el periodo de 1994 a 2006, la mortalidad total de defunciones registradas por fueron 101,172 de las cuales el suicidio ocupó la cuarta posición con $8.7 \%$ de los casos. Agregando García et al. (2006) que los datos estadísticos manifiestan un aumento del 13\% en dos años $y$, de diez veces las muertes por suicidio entre los adolescentes en los pasados 9 años, correspondiendo a un $82 \%$ hombres y el $18 \%$ a mujeres.

En tal sentido Puentes, López y Martínez (2001) mencionan que pese a dicho aumento, el incremento del índice estadístico no fue de forma equitativa para el total de los estados mexicanos: presentando en el año del 2001, las tasas más altas de morbilidad por suicidio los estados de Campeche y Tabasco, registrando alrededor de 9 suicidios por 100,000 habitantes; mientras que las tasas más baja correspondieron a los estados de Chiapas y el Estado de México con 1 suicidio por cada 100,000.
Ante tales cifras Borges et al. (2010) indican que para el 2007 se habían registrado 4,388 muertes por suicidio con una tasa de mortalidad de 4.12 por cada 100,000 habitantes, refiriendo que existe una importante diferencia entre las causas por las cuales se dan los suicidios en los países desarrollados y en desarrollo. Aclarando que en los primeros, el trastorno en relación con el estado de ánimo tienen prioridad; mientras que en los segundos el trastorno asociado con el consumo de sustancias y la falta de control de impulsos, esto en cuanto a los principales trastornos asociados con el suicidio.

Ahora bien, según el Sistema Nacional de Informacion en Salud (SINAIS, 2008) entre las principlaes causas de mortalidad en edad productiva en edades de 15 a 64 años de edad en el 2008 a nivel nacional, se presentaba como la novena causa de mortalidad el suicidio, alcanzando en dicho año la cantidad de 4, 089 defunciones (tabla II). Mientras que el INEGI (2010b) un año despues informa que más de una tercera parte del total de los actos de suicidio, la característica que prevalecieron fueron que por cada mujer se identificaron cinco hombres suicidas de entre 14 y 15 años de edad. El mismo organismo estableció que el 2009 se suicidaron más hombres que mujeres en México, registrando 5,190 muertes por suicidio de los cuales 4,201 fueron hombres y 989 mujeres.

Años más tarde la Revista Nexos (2017) indica que desde 1990 y hasta 2015 se suicidaron en México 104 mil personas, $83 \%$ de ellas fueron hombres, y de éstos, $72 \%$ tenían menos de 44 años de edad. Así, casi 62 mil hombres jóvenes se quitaron la vida en 26 años. Destacando que en términos absolutos, en los noventa se suicidaban en promedio cada año 2,731 personas; en el sexenio de Vicente Fox 3,985 personas; en el sexenio de Felipe Calderón, 5,091 personas; y en los primeros tres años de Enrique Peña Nieto, 6,224 personas (gráfico 1); mientras que para el año del 2016 se registraron un total de 6,370 suicidios (gráfico 2) (SINAIS, 2018; INEGI, 2018). 


\section{Tabla II. Principales causas de mortalidad en edad productiva (de 15 a 64 años), 2008. Nacional}

\begin{tabular}{|c|c|c|c|c|c|}
\hline Orden & Clave CIE 10a. Rev. & Descripción & Defunciones & Tasa $1 /$ & $\%$ \\
\hline & A00-Y98 & Total & 202670 & 292.1 & 100.0 \\
\hline 1 & E10-E14 & Diabetes mellitus & 29554 & 42.6 & 14.6 \\
\hline 2 & $\mathrm{~K} 70, \mathrm{~K} 72.1, \mathrm{~K} 73, \mathrm{~K} 74, \mathrm{~K} 76$ & Cirrosis y otras enfermedades crónicas del hígado & 18086 & 26.1 & 8.9 \\
\hline 3 & $120-125$ & Enfermedades isquémicas del corazón & 14968 & 21.6 & 7.4 \\
\hline 4 & 21 & Accidentes de tráfico de vehículo de motor & 13314 & 19.2 & 6.6 \\
\hline 5 & X85-Y09, Y87.1 & Agresiones (homicidios) & 12689 & 18.3 & 6.3 \\
\hline 6 & $160-169$ & Enfermedad cerebrovascular & 7054 & 10.2 & 3.5 \\
\hline 7 & B20-B24 & $\mathrm{VIH} / \mathrm{SIDA}$ & 4961 & 7.2 & 2.4 \\
\hline 8 & N00-N19 & Nefritis y nefrosis & 4749 & 6.8 & 2.3 \\
\hline 9 & $\mathrm{X} 60-\mathrm{X} 84, \mathrm{Y} 87.0$ & Lesiones autoinfligidas intencionalmente (suicidios) & 4089 & 5.9 & 2.0 \\
\hline 10 & $110-\mid 15$ & Enfermedades hipertensivas & 3363 & 4.8 & 1.7 \\
\hline 11 & C50 & Tumor maligno de la mama & 3275 & 4.7 & 1.6 \\
\hline 12 & J10-J18, J20-J22 & Infecciones respiratorias agudas bajas & 2969 & 4.3 & 1.5 \\
\hline 13 & C53 & Tumor maligno del cuello del útero & 2446 & 3.5 & 1.2 \\
\hline 14 & C16 & Tumor maligno del estómago & 2371 & 3.4 & 1.2 \\
\hline 15 & $\mathrm{~J} 40-\mathrm{J} 44, \mathrm{~J} 67$ & Enfermedad pulmonar obstructiva crónica & 2146 & 3.1 & 1.1 \\
\hline 16 & & Uso de alcohol & 2095 & 3.0 & 1.0 \\
\hline 17 & C91-C95 & Tumor maligno de tráquea, bronquios y pulmón & 2007 & 2.9 & 1.0 \\
\hline 18 & F10, G31.2 & Leucemia & 1968 & 2.8 & 1.0 \\
\hline 19 & C81-C90 & Linfomas y mieloma múltiple & 1822 & 2.6 & 0.9 \\
\hline \multirow[t]{3}{*}{20} & W65-W74 & Ahogamiento y sumersión accidentales & 1773 & 2.6 & 0.9 \\
\hline & R00-R99 & Causas mal definidas & 1761 & 2.5 & 0.9 \\
\hline & & Las demás & 65210 & 94.0 & 32.2 \\
\hline
\end{tabular}

1/ Tasa por 100,000 habitantes

No se incluyen defunciones de residentes en el extranjero

2/ V02-V04 (.1, .9), V09.2-V09.3, V09.9, V12-V14 (.3-.9), V19.4-V19.6, V20-V28 (.3-.9), V29-V79 (.4-.9), V80.3-V80.5, V81.1,

V82.1, V83-V86(.0-.3), V87.0-V87.8, V89.2, V89.9, Y85.0

Las principales causas de mortalidad están basadas en la lista GBD de 165

Fuente: Secretaría de Salud/Dirección General de Información en Salud. Elaborado a partir de la base de datos de defunciones 19792008 INEGI/SS y de las Proyecciones de la Población de México 2005 - 2050, y proyección retrospectiva 1990-2004. CONAPO 2006.

Fuente: SINAIS (2008)

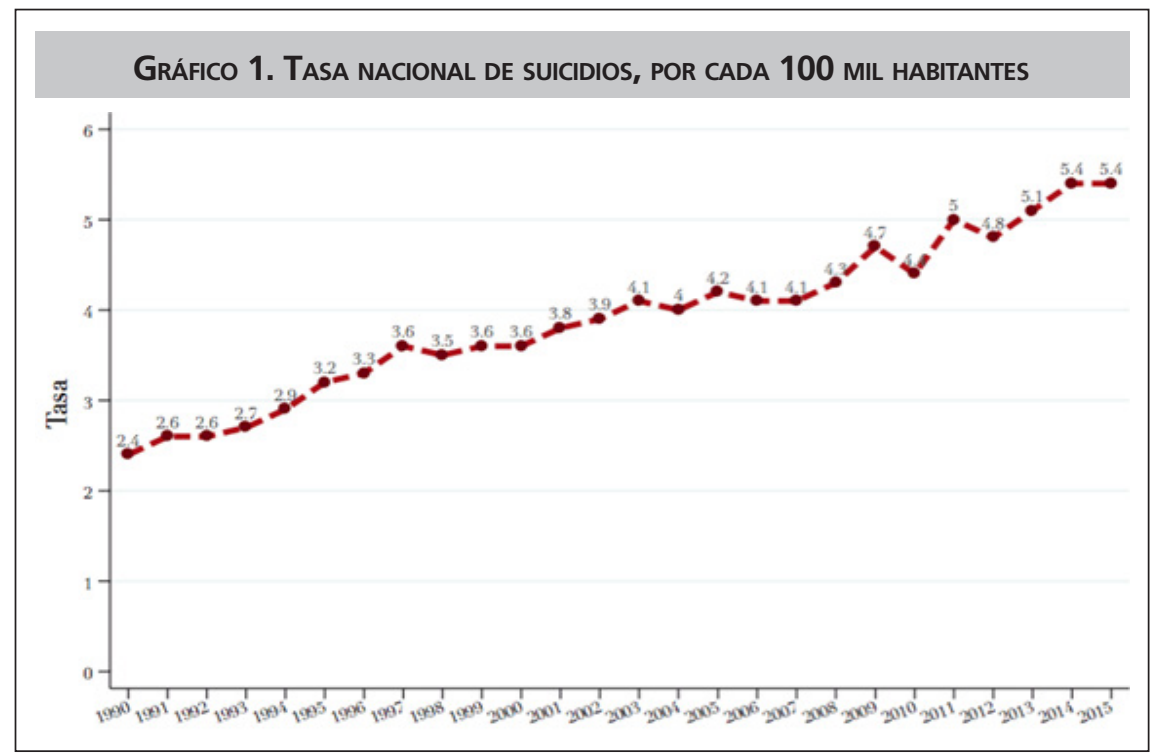

Fuente: SINAIS (2018) 


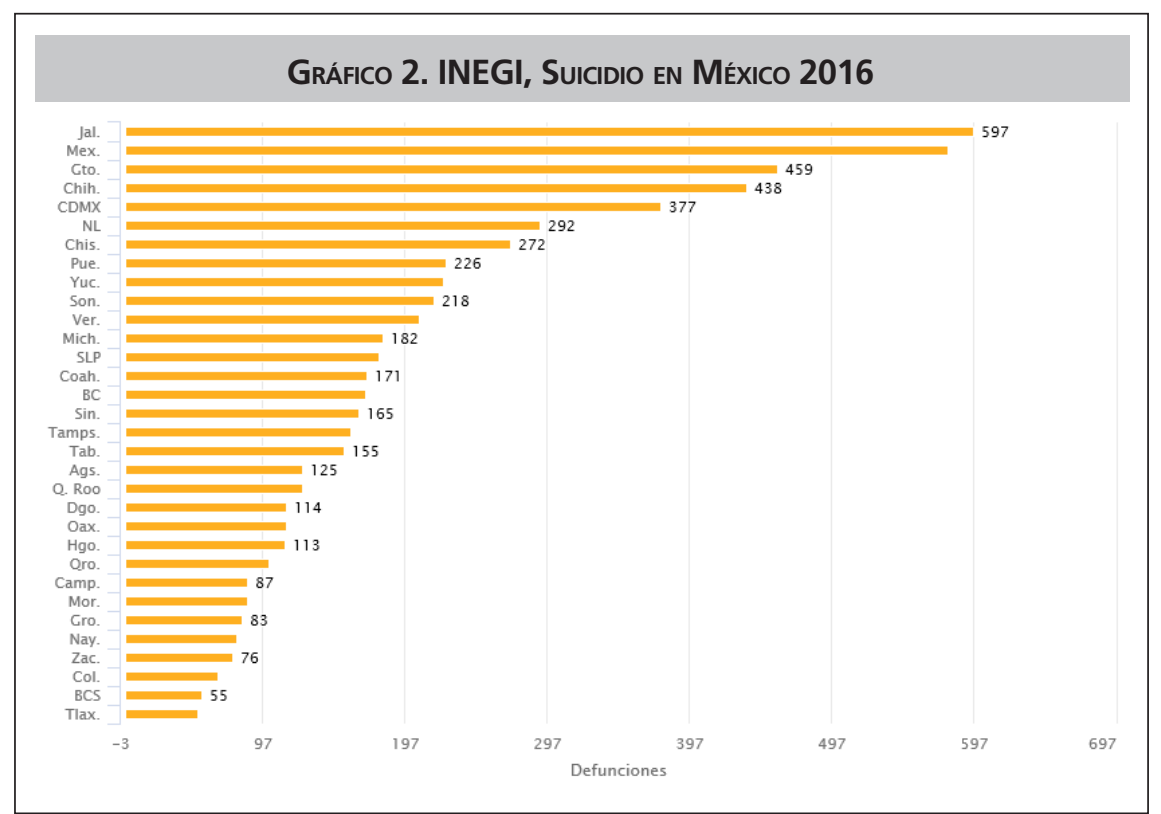

Fuente: INEGI (2018)

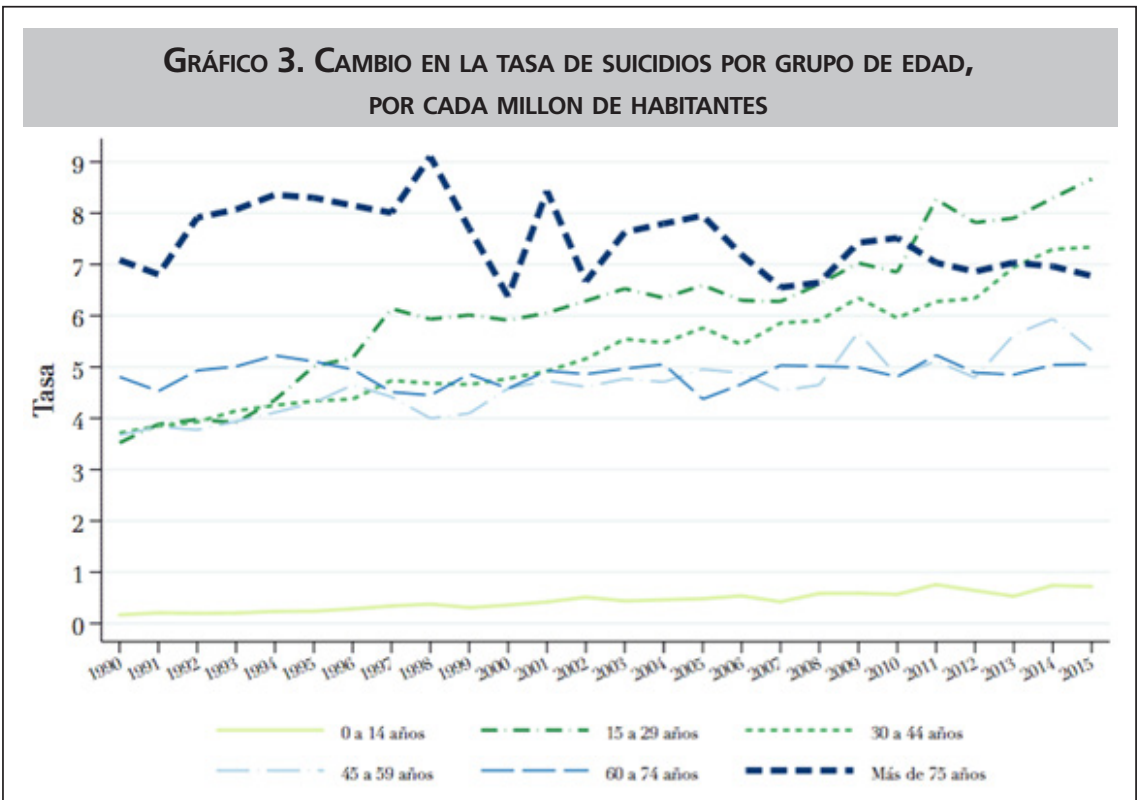

Fuente: SINAIS (2018)

Además, aunque la proporción de adultos mayores a 75 años que se suicidan se ha mantenido constante, es alarmante notar que este grupo es el que ha tenido la tasa más alta de entre cualquier grupo poblacional la mayoría del tiempo, alcanzando a acumular 9.1 suicidios por cada cien mil personas mayores a 75 años durante todo el periodo. Sin embargo, en 2011 las personas entre 15 y 29 años superaron la tasa de los adultos mayores, al igual que las personas entre 30 y 44 años (gráfico 3) (SINAIS, 2018). Agregando la Organización Mundial de la Salud (2012) que, pese a que la tasa de suicidios aumenta con la edad, por ejemplo las personas de 75 o más consuman el suicidio tres veces más que la población joven. Un problema creciente en todo el mundo, es la presencia e incremento de suicidios entre jóvenes de 15 a 25 años de edad o niños de entre 5 a 14 años, como lo expone Saldía (2008) quien sostiene que el suicidio representa en la actualidad un fenómeno creciente en dichos grupos etarios.

\subsection{El suicidio infantil}

Bajo tal panorama García et al. (2006) resaltan que en periodo de 1980 al 2002 los suicidios en México aumentaron un $459 \%$, número que resulta alarmante para la población en general, y no tan sólo, por el aumento de los casos suicidas, sino por la presencia de tales conductas en la población menor de 14 años de edad, revelándose de tal manera, el 
verdadero drama del fenómeno como lo es el suicidio infantil.

La Secretaria de Seguridad Pública (2009) apuntan que del total de suicidios registrados en la ciudad de México, el 3\% corresponde a suicidios de personas menores de 15 años, cifras que se incrementaron en un 39\% de 1995 al 2001. Expresando la propia dependencia que en el 2000 el suicidio en niños con edad escolar de entre 5 y 14 años ocupo el octavo lugar. Mientras que el Sistema Nacional de Información en Salud [SINAIS] reportó que en el 2008 se ubicó a las defunciones por suicidio en dicha población en el $4^{\circ}$ lugar; cifras que reflejan, el aumento en un 50\% en un lapso de 4 suicidios en niños escolares años. Agregando que en la población de edad escolar de 5 a 14 años ocupó el $4^{\circ}$ lugar en México.

Por su parte, el INEGI (2011; 2010a) indica que en el año del 2010 se suicidaron 5,012 personas de los cuales 4,091 son hombres y 921 son mujeres; sobresaliendo los 187 casos suicidas en población de 10 a 14 años. Agregando la Organización Mundial de la Salud (2012) que el suicidio infantil, se encuentra en la actualidad, entre las cinco primeras causas de muertes infantiles, afirmando que entre los principales factores que se asocian a tal desenlace se encuentran: los trastornos mentales como la depresión y los trastornos por consumo de alcohol, factores que incrementan el riesgo suicida —como sucede en Europa y América del Norte-; agregando que el problema es tan dramático y creciente que cada 40 segundos se suicida una persona en el mundo, lo cual expone el fenómeno del suicidio como una de las principales causas de muerte.

En el caso particular de México el SINAIS (2008) registró en el periodo del 2000 al 2008, que las cinco principales causas de muerte en edad escolar de 5 a 14 años: accidentes de tráfico de vehículo de motor 7,471 muertes; leucemia 5,368 muertes; ahogamiento y sumersión accidentales 2,942 muertes; agresiones (homicidios) 2,136 muertes; infecciones respiratorias agudas bajas con 1,801 muertes; apareciendo de forma dramática las lesiones autoinfligidas intencionalmente (suicidios) con 1,038 muertes.

Dicho escenario demuestra cómo el suicidio infantil se materializó como un fenómeno social de creciente impacto, pero lo más relevante y a su vez, alarmarte es identificar como el suicidio se encuentra dentro de las primeras causas de muerte en la población de 5 a 14 años de edad; población que al tiempo que está empezando a vivir su vida, decide privarse de ella. Durkheim (1965) sostiene que el suicidio en la población infantil es extremadamente raro, narrando que en Francia por ejemplo, por cada millón de niños menores de 16 años se produjeron en el periodo de 1861 a 1875, 4.3 suicidios de varones y 1.8 suicidios en niñas. Afirmando que pese a que el suicidio infantil no es frecuente, lo cierto es que el niño, al estar expuesto a diversas causas sociales, lo pueden orillar a tomar dicha resolución.

Sarró y De la Cruz (2000) indican que el suicidio infantil no es tan frecuente antes de los doce años, pero existe, una de las razones para explicar la baja tasa de suicidios en estas edades es la aceptacion de que un niño no vive la muerte como un fin y, por esto, a nivel cognitivo, no puede plantearse el suicidio. Afirmando que un niño a partir de los siete años ya comprende el concepto muerte, pero muchos factores que contribuyen a su entendimiento no han sido evaluados todavia. En tal sentido, Pfeffer (1986) sostiene que la definición de conducta suicida en el niño es similar a la del adulto; pero en este primer caso lo va madurando con edad. Agregando que desde hace 150 años se han ido recolectando observaciones clínicas sobre actos suicidas en niños de 2 a 14 años.

Destacando Orbach, Rosenheim y Hary (1987) que dentro del acto suicida del niño aparecen aspectos similares al del adolescente, como la planificación, los métodos, la depresión y la percepción de la muerte y, sobre todo, el hecho de que en estas edades también puede ser un proceso prolongado y, no un acto manipúlado o impulsivo. Al respecto Castillo y Hernández (2007) argumentan que entre los principales factores para la consumación del acto suicida infantil representa la participación del medio familiar, la cual puede contribuir en el deseo de una persona a quitarse la vida.

Morán (2004) agrega que dichos factores familiares están asociados a trastornos cognoscitivos del estado de ánimo, de identidad, de apego, de desesperanza y de dolor emocional, sin descartar que el infante suicida puede presentar problemas de aprendizaje, cambios de actitudes, o desordenes en el sueño o en otra función cognitiva o motora (Prensalibre, 2013). Nelson y Galas (2004) agregan que los sujetos que tienen dificultades para aprender y a quienes les va mal en la escuela, a menudo sufren de baja autoestima y depresión, atravesando episodios de ideación suicida o consumación del acto (Noticias Terra, 2013).

Mientras tanto, la Secretaria de Seguridad Publica (2009) expone que la ideación suicida puede presentarse en niños que al ser víctimas del maltrato de tipo sexual y posteriormente no sentirse escuchados ni protegidos, tienden a presentar conductas auto-agresivas que pueden llegar al suicidio. Sin embargo, como lo plantea Petrzelová (2013) no es sólo el maltrato sexual lo que puede generar que el infante albergue desesperanza o profunda tristeza por alguna perdida, al grado de atentar contra su propia vida, sino que cualquier tipo de maltrato puede detonar dicho acto. Destacando Acevedo que el castigo infantil frustra e impide un saludable desarrollo 
cognoscitivo, genera experiencias traumáticas en la vida del infante, dando pie a las alteraciones emocionales, inestabilidad familiar y social lo que ubicará al niño dentro de una situación de no encontrar "escapatoria" (2010).

El ser víctima de castigo y malos tratos representa como lo indica Acevedo (2010) un detonador para las autolesiones y las conductas de riesgo; en ocasiones los gritos silenciosos y de ayuda, se materializan no como un exclamo de "auxilio", sino a través de alguna conducta o acción que desvíe la mirada de los "otros", como pueden desde las conductas antisociales del menor (peleas, insultos, desobediencia, mala conducta en la escuela, el bullying, entre otros), hasta la autolesión o el suicidio (Acevedo, 2012; 2013). En tal sentido Catillo et al. (2007) narran que en la ciudad de Guanajuato al realizar un análisis ${ }^{2}$ de 29 de las 42 defunciones por suicidio en la ciudad, descubrieron que el $51.7 \%$ sufrían violencia en casa, particularmente dos de los 4 menores, los cuales recibían maltrato plenamente identificado y plasmando en sus notas póstumas con frases de "no viví tranquilo en esta vida que me daban", " Ya sé que no me quieren espero me avienten pa'l arrollo", "Si me suicide fue porque nunca creen en mi" y "ya me canse" (...) "que tú me regañes yo no tengo apoyo de ustedes" (Diario la VeraDonimicana, 2008, pag. 1).

Por otra parte, Delgado (1997) expone que la mortalidad infantil por administración de agentes químicos se presenta en un 15 a 20\% de los casos, dejando secuelas neurológicas la mayoría de las veces, especialmente cuando los agentes utilizados son los más peligrosos, como la insulina y los hi- drocarburos. Mencionando Karlin (1995) que entre las principales maneras como mueren los niños es debido a las lesiones autoinfligidas o suicidio, sofocación, heridas inducidas, desangramiento, envenenamiento crónico con ipecacuana, envenenamiento crónico phenolphethalein (inducción a la diarrea) e inyección de excrementos y ahorcamientos; derivados en ocasiones por juegos aparentemente inocentes como la práctica del "juego del desmayo" o "choking game"3 (Pijamassurf, 2010). Delgado (1997) agrega que el incremento del suicidio infantil se puede asociar, a la fragilidad de los lazos sociales y familiares, los cuales al estar fracturándose dan como consecuencia las cifras expuestas, o la presencia de las lesiones auto infringidas intencionalmente (suicidio).

Tal escenario revela hasta cierto punto una magnitud del problema, sin embargo como lo declaran Carabaza y Ewald (2007) se pueden presentar datos que no son reportados a alguna dependencia gubernamental, pero que si son registrados por la prensa; lo que revela que la cifra oficial, puede variar considerablemente (tabla III). Delgado (1997) coincide

\begin{tabular}{|ccc|}
\hline \multicolumn{3}{|c|}{$\begin{array}{c}\text { Tabla III. SuICIDIOS Registrados POR LA PGI } \\
\text { EN El EStAdo De CoAHUILA }\end{array}$} \\
2002-2003 \\
Año & Notas publicadas & Reportados \\
\hline 2002 & 47 & 34 \\
\hline 2003 & 66 & 34 \\
\hline Total & 113 & 68 \\
\hline
\end{tabular}

Fuente: Carabaza y Ewald (2007)

\begin{tabular}{|c|c|c|c|c|c|c|c|}
\hline Factores & $\begin{array}{l}\text { Gruhle } \\
(1949)\end{array}$ & $\begin{array}{l}\text { Pokorny } \\
(1974)\end{array}$ & $\begin{array}{c}\text { Gornitz } \\
\text { (1975) }\end{array}$ & $\begin{array}{l}\text { Monk } \\
(1975)\end{array}$ & $\begin{array}{l}\text { Barraclough } \\
\text { (1978) }\end{array}$ & $\begin{array}{l}\text { Baechiler } \\
\text { (1979) }\end{array}$ & $\begin{array}{l}\text { Holinger } \\
\text { (1979) }\end{array}$ \\
\hline Cambios en las condiciones sociales (tabús) & * & & & * & * & * & \\
\hline $\begin{array}{l}\text { Dificultades para establecer el diagnóstico médico-legal } \\
\text { diferencial con otras causas de muerte violenta. }\end{array}$ & & & & & & & * \\
\hline $\begin{array}{l}\text { Carencia de elementos para establecer la causa de } \\
\text { muerte }\end{array}$ & & & & * & & & \\
\hline $\begin{array}{l}\text { Diferencias en las estrategias para establecer causas de } \\
\text { muerte. }\end{array}$ & & * & & & & & * \\
\hline $\begin{array}{l}\text { Cambios y/o diferencias en las definiciones de causas } \\
\text { y/o casos. }\end{array}$ & & & * & & & & * \\
\hline Cambios en las estrategias de registro. & & * & * & & & & * \\
\hline $\begin{array}{l}\text { Falsos testimonios de muerte realizados positivamente } \\
\text { por el médico. }\end{array}$ & & & & & & & * \\
\hline $\begin{array}{l}\text { Falsas declaraciones realizadas por los testigos en } \\
\text { relación a la causa de muerte. }\end{array}$ & & & & * & & & * \\
\hline \multicolumn{8}{|l|}{ Confusión por los efectos colaterales de neurolépticos. } \\
\hline \multicolumn{8}{|l|}{ Certificación de defunciones no confiables. } \\
\hline Inhumaciones clandestinas. & & & & & & & \\
\hline
\end{tabular}

Fuente: Nadelsticher y Gorenc (1985) 


\section{CUADRO 1. FACTORES QUE PUEDEN ESTAR INVOLUCRADOS EN LA MAGNITUD DE LA CIFRA OBSCURA DEL SUICIDIO SEGÚN DIVERSOS AUTORES. $2^{\mathrm{a}}$ PARTE}

\begin{tabular}{|c|c|c|c|c|c|c|c|c|}
\hline Factores & $\begin{array}{l}\text { Wekstein } \\
\text { (1979) }\end{array}$ & $\begin{array}{l}\text { Welz } \\
(1979)\end{array}$ & $\begin{array}{l}\text { Farmer } \\
(1980)\end{array}$ & $\begin{array}{c}\text { Hernrich } \\
(1980)\end{array}$ & $\begin{array}{l}\text { Gorenc } \\
\text { y Kleff } \\
\text { (1981) }\end{array}$ & $\begin{array}{c}\text { Wellhofer } \\
\text { (1981) }\end{array}$ & $\begin{array}{l}\text { Schmidtke } \\
\text { (1981) }\end{array}$ & $\begin{array}{c}\text { Gorenc y } \\
\text { Nadelsticher } \\
\text { (1985) }\end{array}$ \\
\hline Cambios en las condiciones sociales (tabús) & * & & & * & & * & * & * \\
\hline $\begin{array}{l}\text { Dificultades para establecer el diagnóstico médico- } \\
\text { legal diferencial con otras causas de muerte violenta. }\end{array}$ & & & * & & * & & & \\
\hline \multicolumn{9}{|l|}{$\begin{array}{l}\text { Carencia de elementos para establecer la causa de } \\
\text { muerte }\end{array}$} \\
\hline \multicolumn{9}{|l|}{$\begin{array}{l}\text { Diferencias en las estrategias para establecer causas } \\
\text { de muerte. }\end{array}$} \\
\hline $\begin{array}{l}\text { Cambios y/o diferencias en las definiciones de causas } \\
\text { y/o casos. }\end{array}$ & & * & & & * & & & \\
\hline Cambios en las estrategias de registro. & & & & * & & & * & * \\
\hline $\begin{array}{l}\text { Falsos testimonios de muerte realizados } \\
\text { positivamente por el médico. }\end{array}$ & & & & & & * & & \\
\hline $\begin{array}{l}\text { Falsas declaraciones realizadas por los testigos en } \\
\text { relación a la causa de muerte. }\end{array}$ & & & & & & * & & \\
\hline $\begin{array}{l}\text { Confusión por los efectos colaterales de } \\
\text { neurolépticos. }\end{array}$ & & & & & * & & & \\
\hline Certificación de defunciones no confiables. & & & & & & & * & * \\
\hline Inhumaciones clandestinas. & & & & & & & & * \\
\hline
\end{tabular}

Fuente: Nadelsticher y Gorenc (1985)

en que tales datos pueden ser superiores, presentándose otras muertes infantiles no definidas o casos de "muerte súbita", Nadelsticher y Gorenc (1985) en la misma dirección argumentan que, en ocasiones es difícil precisar dichas cifras, debido a la presencia de factores involucrados dentro de la cultura del reporte o registro como pueden ser: los cambios en las condiciones sociales, carencia de elementos para establecer la causa de muerte, los cambios o diferencias en las definiciones de causas y casos, entre otros (cuadro $1,1^{\text {a }}$ parte y $2^{\mathrm{a}}$ parte).

De la Peña (s/f) indica que la conducta suicida involucra diferentes manifestaciones: conductuales, cognitivas y emocionales. Se incluyen dentro de la conductas suicidas las ideas de muerte, las ideas suicidas, las autolesiones, los intentos suicidas y los suicidios consumados. En México, el suicidio es un problema de salud pública, según los registros de la Secretaría de Salud, las tasas de mortalidad por esta causa han aumentado en los últimos años, especialmente en niños y adolescentes. Dichas cifras exponen la necesidad de hacer una comprensión más profunda de la dinámica del suicidio infantil, pues cada vez, se incrementan —como se pudo observar-y se encuentran más evidencias que muestran lo común que se está presentando entre la población infantil, lo cual, no sólo es devastador, si no fatal para los niños y sus familias; pero de manera puntual deja en entredicho el papel de la sociedad frente al cuidado de sus propios hijos (Secretaría de Seguridad Pública, 2009).
Finalmente Sarró y De la Cruz (2000) indican que el interes por esta problemática se objetiva por el aumento de las publicaciones sobre actos de suicidio en la infancia y en la adolescencia, por ejemplo desde 1900 a 1967 se recolectaron 200 libros y artículos relacionados al tema; mientras que la decada de 1980 se incrementaron las investigaciones del tópico, denotando que si bien el fenomeno es inusual, si existe en menores de 12 años; particulamente en poblaciones en edad escolar de 6 a 12 años. Expresando los mismos autores que el suicidio consumado en una tragedia no solo para los familiares y amigos, sino para toda la sociedad en su conjunto; que cimbran a las intituciones y cuestionan a las políticas públicas por lograr intrumentar adecuadamente acciones concretas para evitar este fenómeno; acciones en las cuales todos partipen desde su trinchera.

\subsection{El suicidio infantil y el trabajo social}

Es más que claro que fenomenos como los ya expuestos representan desafios para cualquier ambito, y el trabajo social no es la excepción, disciplina científica que por sus características humanisticas se vincula estrechamente con fenomenos de tal naturaleza. En tal sentido, hoy día se requiere de estrategias de mayor presición, acompañadas de politicas publicas incluyentes, en donde se refleje la participación de todo los actores sociales e institucionales, al igual que profe- 
sionales más habiltados en temas complejos como el suicidio, y particularmente el suicidio infantil. Que por sus características requiere de conocimientos, teorias, metedologias de intervención de mayor puntualidad y especialidad. Pero sobre todo, que el profesional del trabajo social sea sensible y pueda reconocer los sintomas o indicadores de conductas de riesgo, y con ello, desde su trinchera comenzar los procesos de atención. Que pueden ir desde simples, como el acompañamiento del niño en situación critica, o hasta más profesionales como la terapia, o el couching de riesgo.

Recordando que en principio se debe de atender factores como la depresión o ansiedad que que puede estas atravezando el niño, y se debe de cuidar a que aumente sin control. En segundo término, se debe de escuchar con atención, y estar atentos de los tipos de lenguajes que puede estar expresando, incluso los no verbales que son los que nos pueden estar indiando el sentir del niño. Además, de nunca ignorar las amenazas de suicido, tachandolos de melodramas propios de la edad. Y de necesitarlo, buscar ayuda profesional de inmediato. Pasos minimos que todos debemos de habilitarnos para la atencion de fenómenos tan sentidos y extremos como estos.

\section{CONCLUSIONES}

Hablar de suicidio infantil en el contexto contemporáneo alude a situaciones inexorables que necesitan respuesta, más aún si se trata de niñas y niños que ya no encuentran motivos porqué vivir, o seguir adelante; ni mucho menos pueden identificar un rumbo, o aferrarse a algún proyecto de vida. Dicho panorama revela que el suicidio infantil es y será uno de los mayores retos para la sociedad en general, principalmente para la familia y las instituciones educativas, e incluxive para los trabajadores sociales, los cuales requeren de todo el intrumental, estrategias y metodologias para estar a la altura de tal desafio.

El suicidio infantil representa un desafío, independientemente de la óptica con la que se mire, debido a que conforme pasa el tiempo se observan sociedades fragmentadas, cada vez en mayor medida; lo que se verá reflejado en el aumento de la población no sólo en riesgo a desarrollar el proceso de las ideaciones suicidas, sino también a consumarlo. De tal manera, es necesario reconocer que dicha problemática no es competencia únicamente del área médica, psicológica o de la psiquiatría sino que representa un problema multidisciplinario, en donde el trabajo social este presente, y contribuya desde su perfil en la generación de modelos de intervención; en la generación de nuevas investigaciones que permitan ampliar el conocimiento de la temática desde dis- tintas miradas; generar escenarios apropiados que permitan al niño obtener un adecuado desarrollo biopsicosocial; promover la reconstrucción de los tejidos familiares, los cuales contribuyan al desarrollo adecuado del niño (Acevedo, 2015; Organización Mundial de la Salud, 2018).

De igual manera, desde el quehacer del trabajo social se debe de implementar líneas de acción que permitan fomentar cambios de rutina dentro de los grupos familiares, orientados a la recuperación de los valores sociales y familiares; propiciar una cultura de respeto por parte de los medios de comunicación, en donde se traten con ética y respeto a los actores suicidas; involucrar a las instituciones educativas, de manera que puedan generar talleres de superación y motivación personal; contribuir desde las distintas trincheras hacia la estabilidad emocional en el niño a partir de la creación de programas donde se promuevan actividades de interés para ellos; crear grupos de autoayuda en las escuelas, que permitan identificar características de riesgo en sus compañeros, y que sean ellos, los que brinden en un primer momento el apoyo emocional, para después canalizarlo con algún profesional; finalmente propiciar espacios dedicados a la expresión de ideas, sentimientos o sensaciones donde el niño manifieste sus intereses y emociones; pero lo mas importante detonar iniciativas donde los propio niños se sientan parte de la solución. ¡Todos juntos podemos ganar esta batalla! (Acevedo, 2015; Organización Mundial de la Salud, 2018).

\section{REFERENCIAS}

Acevedo, J. (2010). El castigo infantil en México: las prácticas ocultas. México, D.F.: Plaza y Valdés.

Acevedo, J. (2012). ¡Tengo Miedo! Bullying en las Escuelas. México, D.F.: Trillas.

Acevedo, J. (2013). Infierno en casa, hay amores que matan. México, D.F.: Trillas.

Acevedo, J. (2015). Gritos silenciosos, el suicidio infantil. México, D.F.: Trillas

Borges, B. \& Medina, M. (2010). Sistema de Información Científica, Suicidio y Conductas Suicidas en México. Retrospectiva y Situación Actual, vol. 52 (Núm. 04), pp 125129. Recuperado de <http://redalyc.uaemex.mx/redalyd src/inicio/ArtPdfRed.jsp?iCve=10617416005>.

Carabaza, J. \& Ewald, A. (2007). El suicidio, un enfoque desde la comunicación. ¿Por qué y cómo se llega a la desesperanza?. En Petrzelova et al. (2007). Tres miradas sobre el suicidio. México, D.F.: Plaza y Valdés.

Castillo, s/n \& Hernández, s/n (2007). Salud Mental. Maltrato y Suicido Infantil en el estado de Guanajuato. Recupera- 
do de <http://redalyc.uaemex.mx/redalyc/src/inicio/ArtPdfRed.jsp?iCve $=58230307>$.

Centro de Información de las Naciones Unidas (CINU) (2013). Cifras sobre suicidio. Recuperado de <http://www.cinu. mx/eventos/observancia/dia-mundial-para-la-prevencion- $1 />$.

Chávez, s/n, Medina, s/n \& Mancías, s/n (2008). Modelo Psicoeducativo para la Prevención del Suicidio en Jóvenes, vol. 31, (numero 003), pp. 1-10. Recuperado de <http:// redalyc.uaemex.mx/redalyc/src/inicio/ArtPdfRed.jsp?iCve $=58231305>$.

Delgado, A. (1997). Síndrome de Münchausen por Poderes. Niños maltratados. Madrid, España: Díaz de Santos.

De la Peña, F. (s/f). Trata miento para la conducta suicida en México: niños y adolescentes. En R. De la Fuente (s/f). Una Propuesta para la Prevención del Suicidio en México. Mexico, D. F.: Instituto Nacional de Psiquiatría.

Durkheim, E. (1965[2011]). El suicidio. México, D.F.: Editorial Fontamara.

Diario la VeraDonimicana (2008). Niño golpeado por papá se suicida. Recuperado de <http://laveraddominicana.wordpress.com/2008/08/07/nino-golpeado-por-papa-se-suicida/>.

García, C., Contreras, M. \& Orozco, R. (2006). Salud Mental. El suicidio Conceptos Actuales, vol. 29 (Núm. 005), pp. 25-30. Recuperado de <http://redalyc.uaemex.mx/ redalyc/src/inicio/ArtPdfRed.jsp?iCve=58229510 > .

Instituto Nacional de Estadística y Geografía [INEGI] (2005). Estadística de Suicidios en los Estados Unidos Mexicanos,. Recuperado de http://www.inegi.org.mx/

Instituto Nacional de Estadística y Geografía [INEGI] (2008). Defunciones 2008, CONAPO, INEGI/Secretaría de Salud. $D G I S, 2008$. Elaborado a partir de base de datos de defunciones 2008 y CONAPO, 2006. Proyecciones de Población de México 2005-2050. Recuperado de <www.sinais. salud.gob.mx/descargas/xls/Principales CausasxEF>.

Instituto Nacional de Estadística y Geografía [INEGI] (2010a). Estadística de Suicidios en los Estados Unidos Mexicanos. Recuperado de <http://www.inegi.org.mx/prod serv/contenidos/espanol/bvinegi/productos/continuas/sociales/suicidio/2010/suicidios 2010.pdf $>$.

Instituto Nacional de Estadística y Geografía [INEGI] (2010b). Estadística de Suicidios en los Estados Unidos Mexicanos. Recuperado de $<$ http://cuentame.inegi.org.mx/monografias/informacion/coah/poblacion/comotu.aspx?tema=me\&e $=05>$.

Instituto Nacional de Estadística y Geografía [INEGI] (2011). Estadística de Suicidios en los Estados Unidos Mexicanos.
Recuperado de <http://www.inegi.org.mx/prod serv/contenidos/espanol/bvinegi/productos/continuas/sociales/suicidio/2009/suicidios 2009.pdf>.

Instituto Nacional de Estadística y Geografía [INEGI] (2018). El suicidio en México, 2016. Recuperado de http.www. inegi.gob.mx

IndexMundi (2012). Tasa de mortalidad infantil (muertes/1000 nacimientos normales) (2011). Recuperado de $<$ http://www.indexmundi.com/>.

Jueces (16:30). Dato bíblico. Recuperado de <http:// www. biblegateway.com/passage/? search $=$ Jueces $\% 20$ 16:30\&version $=$ NIV $>$.

Jueces (9:54). Dato bíblico. Recuperado de <http://www.biblegateway.com/passage/?search=Jueces\%209:54\&version $=\mathrm{NIV}>$.

Karlin, N. (1995). Münchausen Syndrome by Proxy, Brattleboro Retreat. Psychiatry Review, pp. 35-40. Recuperado de $<$ www.bratretreat.org/brpr./v4n1.html $>$.

Mateo (27:3-10). Dato bíblico. Recuperado de <http:// www. biblegateway.com/passage/? search=Matthew $+27 \% 3 \mathrm{~A} 3-10$ \&version $=\mathrm{NIV}>$.

Morán, R. (2004). Educandos con desordenes emocionales y conductuales. Puerto Rico: Universidad de Puerto Rico.

Maldonado, G. (2003). El Suicidio. Recuperado de $<$ http:// webs.ono.com/aniorte nic/archivos/trabaj_suicidio.pdf>.

Nadelsticher, s/n \& Gorenc, s/n (1985). Cifra obscura del suicidio en México. México D.F: Gobierno de la república mexicana

Nelson, R. \& Galas, J. (2004). Lo que necesitas saber sobre el suicidio. Mi amigo Habla de suicidarse. México D.F.: Panorama Editorial.

Nexos (2017). México tiene un máximo histórico de suicidios. Recuperado de https://parentesis.nexos.com.mx/?p=141

Noticias Terra (2013). Niño se suicida y le desea "bonita vida" a madre. Recuperado de <http://noticias.terra.cl/mundo/nino-se-suicida-y-le-desea-bonita-vida-a-madre,c0b1e022c6d6c310VgnVCM20000099cceb0aRCRD.html>.

Noticias Terra (2013). Niño se suicida en GB porque su madre le quitó el celular. Recuperado de <http://noticias. terra.com.mx/mundo/europa/nino-se-suicida-en-gb-porque-su-madre-le-quito-el-celular, 54f77b2a7e4dc310VgnVCM20000099cceb0aRCRD.html>.

Ocampo, R., Bojorquez, I. \& Cortés, M. (2009). Consumo de sustancias y suicidios en México: resultados del sistema de vigilancia epidemiológica de las adicciones, 1994-2006", Salud pública Méx vol.51 No.4, pp.125-130

Organización Mundial de la Salud [OMS] (2012). Estadísticas del suicidio. Recuperado de <http://www.who.int/men- 
tal health/prevention/suicide/suicideprevent/es/index. html>.

Organización Mundial de la Salud [OMS] (2014). Prevención del suicidio un imperativo global, OMS. Washington, DC: Organización Panamericana de la Salud [OPS].

Organización Mundial de la Salud [OMS] (2018). Prevención del suicidio (SUPRE). Recuperado de http://www.who.int/ mental health/prevention/suicide/suicideprevent/es/

Orbach, I., Rosenheim, E. \& Hary, E. (1987), Some aspects of cognitive functioning in suicidal chilndren, Journal of the American Academy of Child and Adolescent Psychiatry, 26, 2, pp.181-185

Petrzelova, M. (2013). Abuso sexual y el silencio que lo rodea. México, D,F.: Plaza y Valdés.

Prensalibre (2013). Niño se suicida en Teculután. Recuperado de $\quad<$ http://www.prensalibre.com.gt/zacapa/Nino-suicida-Teculutan 0 913108719.html>.

Pijamassurf (2010). La práctica del "juego del desmayo" o "choking game". Recuperado de <http://pijamasurf. com/2010/06/el-juego-de-los-ninos-suicidas-ahorcadoses-mejor/>.

Publico ES (2008). Un niño de 12 años se suicida en un centro de menores. Recuperado de <http://www.publico.es/ espana/180260/un-nino-de-12-anos-se-suicida-en-uncentro-de-menores $>$.

Puentes, E., López, L. \& Martínez, T. (2001). La mortalidad por suicidios: México 1990-2001 (Mortality from suicides: Mexico, 1990-2001), Revista Panamericana de Salud Pública, pp.65-70.

Pfeffer, C.R. (1986). The suicidal child. Nueva York: Guilford Press

Quintanar, F. (2007). Definición y Naturaleza. La Conducta Suicida y el Suicidio; Comportamiento Suicida. México, D.F.: Pax editorial.

Reyes, L.A. (1991). Curso Fundamental de Tanatología: suicidio. México, D.F: Fundamentos de tanatología

Sarró, B. \& De la Cruz, C. (2000). Los suicidios. Madrid, España: Ediciones Martinez Roca, S.A.

Saldía, C. (2008). Saldía, San Carlos niños con ideas suicidas. Recuperado de <http://www.sancarlosaldia.com/noticias/ notas-generales/ninos-con-ideas-suicidas-2.html>.

Secretaria de Salud (2007). Programa Nacional de Salud 2007-2012. Por un México sano: construyendo alianzas para una mejor salud. Recuperado de <http://new.paho. org/hq/dmdocuments/2010/Politicas Nacionales Salud-Mexico 2007-2012.pdf>.
Secretaría de Seguridad Pública, Gobierno Federal (2009). Acciones para evitar la revictimización del niño víctima del delito. Recuperado de <http://www.gobernacion. gob.mx/work/models/SEGOB/Resource/689/1/images/ TOMOI 1E.PDF>.

Sistema Nacional de Información en Salud (SINAIS) (2008). Principales causas de mortalidad en edad escolar (de 5 a 14 años). Recuperado de <http://www.sinais.salud.gob. $\mathrm{mx} /$ basesdedatos/estandar.html>.

Sistema Nacional de Información en Salud (SINAIS) (2018). Tasa nacional de suicidios, por cada 100 mil habitantes. Recuperado de http://www.sinais.salud.gob.mx

Sinembargo.mx (2013). Llaman a fortalecer estrategias contra el suicidio en México. Recuperado de <http://www. sinembargo.mx/31-07-2013/706218>.

1Samuel $(31: 4,5)$. Pasaje bíblico. Recuperado de $<\underline{\text { http:// }}$ www.biblegateway.com/passage/? search $=1 \% 20$ Samuel\%2031:\%204,5\&version=NIV>.

\section{NOTAS :}

1. ABATE. Se daba el nombre de abate en España, Francia, Italia y aun en otros países a una persona que no teniendo por lo común más que ordenes menores católicas, vestía de clérigo a la romana.

2. Trabajando un modelo de estudio llamado autopsia psicológica, mediante la reconstrucción de la vida de los occisos, apoyados en las aportaciones de sus familiares cercanos y notas póstumas, diarios y cualquier otra evidencia que pueda ser útil para dicho proceso.

3. El choking game o juego del desmayo, también conocido como california dreaming, black out game, airplaning o tantos otros nombres (se tienen antecedente de él desde 1890). En lo que va desde abril del 2010 , se han suicidado siete adolescentes entre doce y diecinueve años; juego practicado por miles de niños, en EEUU y México, tiene la característica de que es muchas veces en grupo y tus compañeros te "salvan de morir" aunque muchas veces sea demasiado tarde. En el caso de los chicos la autoasfixia fue en soledad, se ahorcaron con una corbata o una bufanda azul solos, encerrados y aislados, tres de ellos iban al mismo colegio, sin embargo hubo además alrededor de diez intentos frustrados. Para jugarlo se exclama la frase de "...Satanás entró y hoy te tienes que matar, yo te voy a decir cuando..." 